\title{
A systematic review of screening instruments for depression for use in antenatal services in low resource settings
}

\author{
Genesis Chorwe-Sungani ${ }^{1,2^{*}}$ and Jennifer Chipps ${ }^{1,3}$
}

\begin{abstract}
Background: In low resource settings, short, valid and reliable instruments with good high sensitivity and specificity are essential for the screening of depression in antenatal care. A review of published evidence on screening instruments for depression for use in antenatal services in low resource settings was conducted. The aim of this review was to appraise the best available evidence on screening instruments suitable for detecting depression in antenatal care in low resource settings.

Methods: Searching, selection, quality assessment, and data abstraction was done by two reviewers. ScienceDirect, CINAHL, MEDLINE, PubMed, SABINET and PsychARTICLES databases were searched using relevant search terms. Retrieved studies were evaluated for relevancy (whether psychometric data were reported) and quality. Data were synthesised and sensitivity and specificity of instruments were pooled using forest plots.

Results: Eleven articles were included in the review. The methodological quality ranged from adequate to excellent. The review found 7 tools with varying levels of accuracy, sensitivity and specificity, including the Edinburgh Postnatal Depression Scale, Beck Depression Index, Centre for Epidemiologic Studies Depression Scale 20, Hamilton Rating Scale for Depression, Hopkins Symptoms Checklist-25, Kessler Psychological Distress Scale and Self-Reporting Questionnaire. The Edinburgh Postnatal Depression Scale was most common and had the highest level of accuracy (AUC $=.965)$ and sensitivity.
\end{abstract}

Conclusion: This review suggests that the Edinburgh Postnatal Depression Scale can be a suitable instrument of preference for screening antenatal depression in low resource settings because of the reported level of accuracy, sensitivity and specificity.

Prospero registration: CRD42015020316.

Keywords: Depression, screening instrument, antenatal, EPDS, Low resource setting

\section{Background}

Depression is a major health problem affecting pregnant women in low resource settings $[1,2]$ with high prevalence rates of antenatal depression (10.7 to 47\%) [1-4]. Antenatal depression can lead to poor uptake of antenatal care, adverse birth outcomes [3] and is a risk factor for postnatal depression [5]. Routine screening for antenatal depression is essential for early identification of pregnant

\footnotetext{
* Correspondence: genesischorwe@kcn.unima.mw

${ }^{1}$ School of Nursing, University of the Western Cape, Private Bag X17, Bellville 7535, South Africa

${ }^{2}$ Kamuzu College of Nursing, University of Malawi, Blantyre, Malawi Full list of author information is available at the end of the article
}

women with depressive symptoms [6] and routine antenatal contacts with health providers provide opportune times for assessing, preventing and treating depression during pregnancy [7].

There are however some challenges in these settings as many women may be ashamed to speak about depression as there is a cultural expectation of pregnancy happiness. In addition, these settings are understaffed, lack consultation rooms, have heavy workloads with high midwife to pregnant woman ratios. Midwives commonly have limited consultation time to explore depressive symptoms or risk factors and often lack guidelines or tools for assessing psychosocial status of pregnant 
women [8]. In this setting, screening instruments suitable for the early detection of depression must be effective in the identification of individuals who are cases and those who are not [9]. Suitable instruments must therefore demonstrate both high sensitivity and specificity [9].

Many validation studies for depression screening tools have previously been conducted in high income countries (HICs) whose cultures and socio-economic context differ from those in low resource settings. Due to a concern about the variation of performance of screening tools in different populations and settings [10] and with the aim of identifying a tool suitable to be recommended for use in antenatal services in low resource settings, a systematic review of instruments for screening depression in antenatal care in low resource settings was conducted.

\section{Methods}

The Standards for the Reporting of Diagnostic Accuracy Studies (STARD) guidelines were used to conduct the review [10].

\section{Search process}

A limited search of the Cumulative Index of Nursing and Allied Health Literature (CINAHL) and Medline was undertaken to identify relevant keywords contained in the title, abstract, and subject descriptors. Search terms and synonyms were then identified for use in searching different databases for screening studies conducted in antenatal clinics in low resource settings. Low resource settings refer to settings where health care systems do not meet the minimum standards set by the World Health Organisation (WHO) or any other quasigovernmental organisation [11]. In this review, low resource settings were defined as health care settings synonymous with those found in low income and lower middle income countries as defined by World Bank [12] and some health care settings in upper middle income countries (UMICs), such as South Africa, where disparities in the public health infrastructure or supplies or human resources [13] are found. Some articles from low resource settings are not indexed to indicate that they are reporting about health outcomes or disparities for under-served populations in low resource settings [14] and the term, 'low resource settings', was not included in the search terms but applied manually at the article review stage. Date limits were set from 2000 to 2015 in anticipation that a wider period to be searched will yield many relevant studies with recent evidence. Detailed search terms are supplied in Table 1.

The following databases were searched: ScienceDirect, CINAHL, MEDLINE, PubMed, SABINET and PsychARTICLES and results were imported into Endnote. Reference lists of key articles identified were hand searched to identify further relevant articles. Manual searches of indexes and "grey" literature databases were not carried out. The preliminary searches were conducted between August and September 2015 and the final search was done on 4th September 2015.

\section{Review process, selection and data extraction}

After the initial search, duplicates and irrelevant articles (conferences, congresses, editorials, commentaries, reviews, news, old) in the Endnote database were removed and the search data were exported to Excel. Articles for review were then selected in three phases.

\section{Abstract and title screening}

In this phase, the reviewers scanned the identified titles and abstracts independently and indicated in the Excel database which articles were relevant. Where the abstract did not provide enough information or the reviewers were unsure, the full text articles were reviewed and agreement reached between the reviewers on the inclusion or exclusion of the article. A kappa statistic was calculated to assess the level of agreement for eligibility for inclusion at this stage.

\section{Screening based on PICOS criteria}

The second phase of selection consisted of a review of articles by applying and extracting the PICOS criteria: Participants $(\mathrm{P})$ (pregnant women at any stage of pregnancy attending antenatal care), Index test (I) (Screening instrument), Comparator test $(\mathrm{C})$ (gold standard- psychiatric assessment), Outcome measures $(\mathrm{O})$ (psychometric properties of screening instrument) and study setting (S) (low resource settings). In this phase, articles from HICs were excluded. Full text articles from UMICs were reviewed and included if the study setting was a public health setting and the studies were located in low resource settings where disparities in the public health infrastructure or supplies or human resources in the services were adequately described.

\section{Article review}

In the third phase, full texts of the articles were reviewed for reported validity of one or a combination of depression screening instruments (sensitivity, specificity, area under curve $[\mathrm{AUC}]$ ) and whether a gold standard was present. The articles were independently examined by the reviewers to confirm inclusion. The gold standard was set as a formal diagnostic psychiatric assessment of depression as the most accurate test to detect the presence or absence of depression [15]. Psychiatric diagnostic assessment of depression included the use of the Structured Clinical Interview for DSM-IV (SCID), the Mini-International Neuropsychiatric Interview (MINI), Composite International Diagnostic Interview (CIDI), International Classification of Diseases version 10 (ICD-10) or the Diagnostic 
Table 1 Search terms

\begin{tabular}{|c|c|}
\hline Data base & Terms used \\
\hline \multirow[t]{4}{*}{ ScienceDirect } & ALL ("screening instruments" OR "screening tools" OR "screening scale") and ALL (depression AND antenatal). \\
\hline & $\begin{array}{l}\text { ALL ("screening instruments" OR "screening tools" OR "screening scale") and ALL (depression AND pregnancy OR prenatal) } \\
\text { AND LIMIT-TO (topics, "woman, patient, depression, depression scale, pregnancy, mental health, depressive symptom, } \\
\text { health care, maternal, adolescent, health"). }\end{array}$ \\
\hline & $\begin{array}{l}\text { ALL (EPDS or CESD-10 or HSCL or K-6 or K-10 or SRQ or PHQ or GHQ) and ALL (depression AND antenatal) AND LIMIT-TO } \\
\text { (topics, "woman, pregnancy, obstet gynecol, depression scale, depression, health, patient, maternal, depressive symptom, } \\
\text { mental health"). }\end{array}$ \\
\hline & $\begin{array}{l}\text { ALL ("screening instruments" OR "screening tools" OR "screening scale") and ALL (depression or "depressive disorder" AND } \\
\text { antenatal or prenatal) }\end{array}$ \\
\hline \multirow[t]{5}{*}{ CINAHL } & TI screening AND TI depression AND TI pregnancy \\
\hline & screening AND depression AND pregnancy AND LIMIT-TO (research article) \\
\hline & screening tools AND depression AND antenatal \\
\hline & epds validity AND depression AND antenatal \\
\hline & $\begin{array}{l}\text { TI Edinburgh postnatal depression scale OR TI Hopkins symptom checklist OR TI self-report questionnaire OR TI center } \\
\text { for epidemiological studies depression scale OR TI patient health questionnaire OR TI general health questionnaire OR } \\
\text { TI beck depression inventory OR TI whooley questions AND TI antenatal AND LIMIT-TO (research article) }\end{array}$ \\
\hline \multirow[t]{4}{*}{ MEDLINE } & TX depression AND TX screening tools AND pregnant women \\
\hline & TI screening test AND TI antenatal depression \\
\hline & TX depression AND TX screening AND TX pregnant women \\
\hline & TI prenatal depression AND TI screening \\
\hline \multirow[t]{3}{*}{ Pubmed } & (((("screening instruments") OR "screening tools") OR "screening scales") AND depression) AND antenatal \\
\hline & ((screening[Title]) AND depression[Title]) AND antenatal[Title] \\
\hline & (((screening[Title]) AND depression[Title]) AND pregnancy[Title]) \\
\hline \multirow[t]{2}{*}{ SABINET } & (alltext:(depression AND screening)^20 AND alltext:(antenatal)^20) \\
\hline & (alltext:(depressive AND disorder AND screening)^20 AND alltext:(pregnant AND women)^20) \\
\hline PsychARTICLES & depression AND screening AND pregnancy \\
\hline
\end{tabular}

and Statistical Manual of Mental Disorders version 4 (DSM-IV) by a psychiatrist to assign a diagnosis. The MINI and SCID are compatible with DSM-IV and have sensitivity/specificity above minimum acceptable level $(.8 / .8)$ for structured interviews which are used as gold standards [16]. Instruments that are routinely used for depression screening such as Edinburgh Postnatal Depression Scale (EPDS) or other nonconventional psychiatric assessment instruments were not considered as gold standards.

Eligibility for full article review, assessment of study characteristics, and relevant data extraction was conducted using a review tool in Excel that included the PICOS criteria and the confirmation of the presence of psychometrics and a gold standard. For each eligible study the reviewers extracted information concerning: author, country of study, sample, gold standard, screening instrument, Area under the Curve (AUC), sensitivity (Se) and specificity (Sp). All results were subject to double data entry.

\section{Assessment of methodological rigour}

The Quality Assessment of Diagnostic Accuracy Studies (QUADAS) [17] was used by both reviewers to assess the psychometric quality of the final selected articles. The QUADAS has 14 items with three possible responses 'Yes', 'No' and 'Unclear'. In the QUADAS, the target condition was depression during pregnancy, the index test was a screening instrument used to screen for depression, and the reference standard was the gold standard against which the index test was validated. The QUADAS items measure the variability of study samples (items 1-2), methodological rigor and bias (items $3-7,10-12$ and 14), and the quality of reporting methodology (items 8, 9 and 13). The scoring of QUADAS is not standardised [18] but studies were categorised as 'excellent' (11 to 14 items), 'good' (9 to 10 items), 'adequate' (6 to 8 items), 'poor' ( 4 to 5 items) or 'unacceptable' ( 0 to 3 items) based on the number of items that were answered 'Yes' [17].

\section{Analysis}

Descriptive data extraction and presentation was done to compare screening instruments' psychometrics data in a between-study literature analysis [19]. A meta-analysis was conducted using REVMAN by pooling individual and all instruments sensitivity and specificity data to show the 
pooled ability of the screening instruments to identify depression. Upper and lower confidence intervals (95\%) for sensitivity and specificity of screening instruments were calculated.

\section{Results}

\section{Search and review results}

The electronic search yielded 3666 published articles (Fig. 1). Eleven (11) additional articles were sourced from authors on ResearchGate and reference lists of full text articles resulting in a total number of 3677 published articles. A total of 1676 duplicates were removed leaving 2001 articles. Irrelevant articles consisting of conferences, congresses, editorials, commentaries, reviews, news and old articles $(\leq 1999)$ were removed ( $n=1750)$, leaving 251 articles. The 251 articles which remained were then screened for relevancy by the reviewers using the PICOS criteria, excluding a further 210 articles [Participants $(n=133)$, Outcome $(n=21)$ and HICs articles $(n=28)$ ], leaving 41 articles (38 primary research studies and 3 systematic reviews). The reviewers' ratings were in agreement with a Kappa $=.97$.

The systematic reviews $(n=3)$ were excluded after being screened for relevancy for inclusion in this review. One systematic review [20] focused on the efficacy of antenatal group interventions aimed at reducing postnatal depression in at risk women. This systematic review did not report any validity data of the depression screening instruments and thus was excluded. The second systematic review by Akena and colleagues [21] examined the accuracy of depression screening instruments validated in general health settings in low and middle income countries (LMICs). This systematic review included three studies conducted in antenatal settings $[4,22,23]$ which also had been identified as part of the 38 articles for primary studies in our review. The third systematic review focused on the reliability and validity of instruments for screening perinatal depression in African settings [24]. This systematic review included eight articles for studies which were conducted in antenatal settings of which four $[3,25,26]$ were included in the 38 primary articles in our review. The other four articles [27-30] were published before 2000 and were excluded due to the time limits of the search terms. Further review of the full texts of the 38 articles showed that two pairs of articles $[25,31]$ and $[3,26]]$ reported the same data from two different studies and one article from each pair was retained resulting in 36 articles included for further review.

\section{Selected studies for full text review $(n=36)$}

The study characteristics of the 36 selected studies for further review are provided in Table 2 . The majority of the studies were published between 2010 and 2015 and only one study was published in a nursing journal. Most of the articles $(n=18)$ were cross sectional prevalence studies and five $(n=5)$ were psychometric validation studies measuring reliability and validity of screening instruments. In reviewing these studies for reported psychometrics of sensitivity, specificity, Area under the curve and the relevant gold standards, two studies $[32,33]$ were excluded (no gold standard as defined by this study) and a further 23 studies were excluded due to inadequate reporting of psychometrics.

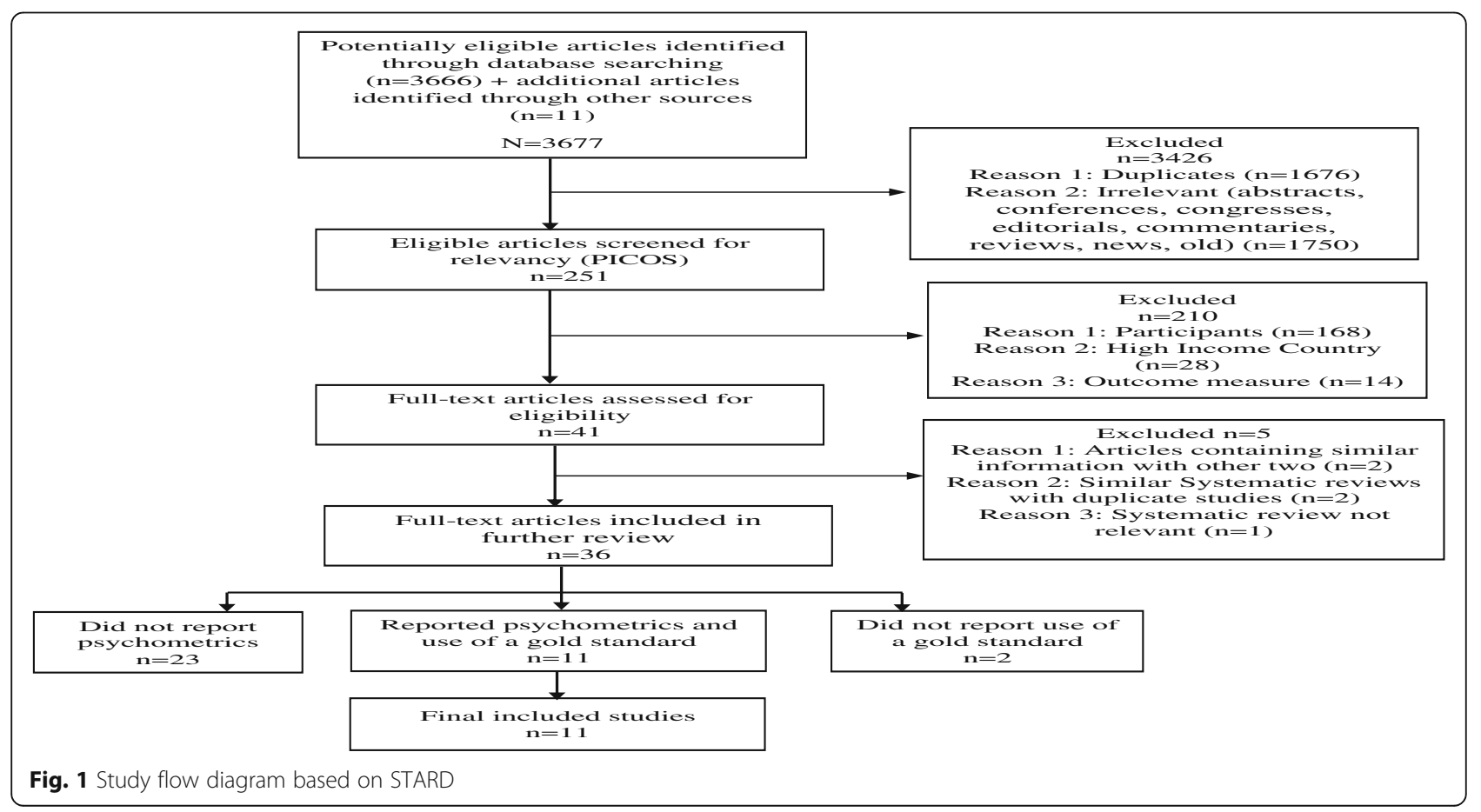


Table 2 Characteristics of 36 studies considered for review

\begin{tabular}{|c|c|c|}
\hline Characteristics & $n=36(100 \%)$ & $n=11(100 \%)$ \\
\hline \multicolumn{3}{|l|}{ Year of publication } \\
\hline $2000-2009$ & 12(33.3) & $3(27.3)$ \\
\hline 2010-2015 & $24(66.7)$ & $8(72.7)$ \\
\hline \multicolumn{3}{|l|}{ Upper Middle Income Country } \\
\hline Brazil & $7(19.4)$ & $2(18.2)$ \\
\hline China & $1(2.8)$ & $0(0)$ \\
\hline Iran & $1(2.8)$ & $0(0)$ \\
\hline Jamaica & $1(2.8)$ & $0(0)$ \\
\hline Peru & $2(5.6)$ & $0(0)$ \\
\hline South Africa & $6(16.7)$ & $2(18.2)$ \\
\hline Thailand & $1(2.8)$ & $0(0)$ \\
\hline Turkey & $2(5.6)$ & $0(0)$ \\
\hline Mexico & $3(8.3)$ & $2(18.2)$ \\
\hline \multicolumn{3}{|l|}{ Lower Middle Income Country } \\
\hline India & $1(2.8)$ & $1(9.1)$ \\
\hline Pakistan & $2(5.6)$ & $1(9.1)$ \\
\hline Sri Lanka & $1(2.8)$ & $0(0)$ \\
\hline \multicolumn{3}{|l|}{ Low Income Country } \\
\hline Malawi & $2(5.6)$ & $1(9.1)$ \\
\hline Tanzania & $4(11.1)$ & $1(9.1)$ \\
\hline Nepal & $1(2.8)$ & $0(0)$ \\
\hline Uganda & $1(2.8)$ & $1(9.1)$ \\
\hline \multicolumn{3}{|l|}{ Study type } \\
\hline Validation & $5(13.9)$ & $5(45.5)$ \\
\hline Epidemiological & $4(11.1)$ & $0(0)$ \\
\hline Cross sectional & $18(50)$ & $4(36.3)$ \\
\hline Randomized controlled trial & $3(8.3)$ & $1(9.1)$ \\
\hline Descriptive & $1(2.8)$ & $0(0)$ \\
\hline Prospective & $3(8.3)$ & $1(9.1)$ \\
\hline Ethnography & $1(2.8)$ & $0(0)$ \\
\hline Naturalistic & $1(2.8)$ & $0(0)$ \\
\hline \multicolumn{3}{|l|}{ Journal type } \\
\hline Medicine & 33(91.6) & $11(100)$ \\
\hline Nursing & $1(2.8)$ & $0(0)$ \\
\hline Multidisciplinary & $1(2.8)$ & $0(0)$ \\
\hline Social and behavioural sciences & $1(2.8)$ & $0(0)$ \\
\hline Se, Sp, AUC, Gold standard reported & 11(30.6) & $11(100)$ \\
\hline
\end{tabular}

$\overline{A U C}$ area under curve, Se sensitivity, $S p$ specificity

One third of the articles $(n=11)$ reported psychometrics and a gold standard and met the final selection criteria for inclusion in the review (Table 2).

Findings from studies for inclusion in review $(n=11)$ All 11 articles were published in medical journals, mostly from 2010 onwards $(n=8)$. A number of articles were validation studies $(n=5)$ that reported psychometrics (reliability and validity). There were also 4 cross sectional prevalence studies $(n=4)$, one prospective study and one randomised trial. These last-mentioned 6 studies generally reported on prevalence of prenatal depression and risk factors but included psychometric properties of the screening instruments. All the screening instruments reported in the selected articles were adapted by translating them to local languages in each setting.

\section{Quality of reviewed studies}

All 11 articles were rated for quality by both reviewers. Overall the quality was satisfactory with six articles $[1,23,25,34-36]$ rated as excellent, three [37-39] good and two [3, 4] adequate. All the articles clearly described the selection criteria for the sample and reported the index test as independent of the gold standard. All articles, except one [39], regardless of overall quality, used random samples. The two articles rated as 'adequate' $[3,4]$ did not sufficiently report the execution of a gold standard and it was difficult to ascertain whether individuals who administered index tests or gold standards were blinded to each other's results. Articles with 'excellent' quality were the psychometric validation studies and the randomised controlled trial.

\section{Screening instruments used in antenatal care in low resource settings}

The articles included seven $(n=7)$ screening tools, namely the Beck Depression Index (BDI), Centre for Epidemiologic Studies Depression Scale (CES-D)-20, Edinburgh Postnatal Depression Scale (EPDS), Hamilton Rating Scale for Depression (HAM-D), Hopkins Symptoms Checklist (HSCL)-25, Kessler Psychological Distress Scale (K-10) and Self-Reporting Questionnaire (SRQ) that were used for screening antenatal depression in low resource settings (Table 3). The BDI and HAM-D are not normally used for diagnostic purposes or screening purposes but to estimate the severity of depression for the past 3 or 7 days. EPDS was designed for use in postnatal period and it has been investigated for antenatal use as well.

Seven studies $(n=7)$ used a single screening instrument while four $(n=4)$ used a combination of two or three instruments. The EPDS was the most widely used instrument ( 8 studies), followed by the BDI and K-10 (2 studies each). The MINI was the most widely used gold standard being used in five of the 11 studies. In assessing the accuracy of screening instruments in detecting depression among pregnant women, an AUC score range is classified as low (.500 to .700), moderate (>.700 to .900) and high (>.900) [40]. The EPDS had the highest level of accuracy (AUC $=0.965)$ while K-10 had the lowest level of accuracy $(A U C=.660)$. The BDI, CES-D, HAM-D, HSCL-25 and SRQ had moderate accuracy with AUC ranges from .820 
Table 3 Results of included studies $(n=11)$

\begin{tabular}{|c|c|c|c|c|c|c|c|c|}
\hline Author & $\begin{array}{l}\text { Country } \\
\text { of study }\end{array}$ & Type of study & Sample (n) & $\begin{array}{l}\text { Gold } \\
\text { standard }\end{array}$ & $\begin{array}{l}\text { Screening } \\
\text { Instrument }\end{array}$ & $\begin{array}{l}\text { AUC } \\
(95 \% \mathrm{Cl})\end{array}$ & $\mathrm{Se}$ & $S p$ \\
\hline Adewuya et al. (2006) [25] & Nigeria & Validation study & $\begin{array}{l}182 \text { pregnant women } \\
(32-36 \text { weeks) }\end{array}$ & $\mathrm{MINI}$ & EPDS & .965 & .867 & .915 \\
\hline $\begin{array}{l}\text { Alvarado-Esquivel et al. } \\
\text { (2014a) [36] }\end{array}$ & Mexico & Validation study & $\begin{array}{l}158 \text { adult pregnant } \\
\text { women ( } 2-9 \text { months) }\end{array}$ & DSM-IV & EPDS & .810 & .757 & .744 \\
\hline $\begin{array}{l}\text { Alvarado-Esquivel et al. } \\
\text { (2014b) [37] }\end{array}$ & Mexico & Validation study & $\begin{array}{l}120 \text { teenage pregnant } \\
\text { women ( } 3-9 \text { months) }\end{array}$ & DSM-IV & EPDS & .890 & .704 & .849 \\
\hline \multirow[t]{3}{*}{ e Couto et al. (2015) [1] } & \multirow[t]{3}{*}{ Brazil } & \multirow[t]{3}{*}{ Validation study } & \multirow{3}{*}{$\begin{array}{l}247 \text { pregnant women } \\
\text { (2nd trimester) }\end{array}$} & \multirow[t]{3}{*}{$\mathrm{MINI}$} & EPDS & .850 & .816 & .733 \\
\hline & & & & & BDI & .900 & .820 & .846 \\
\hline & & & & & HAM-D & .860 & .877 & .746 \\
\hline \multirow[t]{2}{*}{ Fernandes et al. (2011) [4] } & \multirow[t]{2}{*}{ India } & \multirow[t]{2}{*}{ Cross sectional study } & \multirow{2}{*}{$\begin{array}{l}194 \text { pregnant women } \\
\text { (3rd trimester) }\end{array}$} & \multirow[t]{2}{*}{$\mathrm{MINI}$} & EPDS & .950 & 1.00 & .849 \\
\hline & & & & & $\mathrm{K}-10$ & .950 & 1.00 & .813 \\
\hline Kaaya et al. (2002) [23] & Tanzania & Randomized controlled trial & $\begin{array}{l}903 \text { HIV positive pregnant } \\
\text { women ( } 8-26 \text { weeks) }\end{array}$ & SCID & HSCL-25 & .860 & .890 & .800 \\
\hline \multirow[t]{2}{*}{ Martins et al. (2015) [39] } & \multirow[t]{2}{*}{ Brazil } & \multirow[t]{2}{*}{ Cross sectional study } & \multirow{2}{*}{$\begin{array}{l}807 \text { adolescent pregnant } \\
\text { women ( } 2 \text { nd trimester) }\end{array}$} & \multirow[t]{2}{*}{$\mathrm{MINI}$} & EPDS & .890 & .811 & .827 \\
\hline & & & & & BDI & .870 & .867 & .738 \\
\hline Natamba et al. (2014) [35] & Uganda & Cross sectional study & $\begin{array}{l}123 \text { [36 HIV positive and } \\
87 \text { HIV negative pregnant } \\
\text { women] (10-26 weeks) }\end{array}$ & MINI & CES-D-20 & .820 & .727 & .785 \\
\hline Rochat et al. (2013) [3] & South Africa & Cross sectional study & $\begin{array}{l}109 \text { [ } 49 \text { HIV positive and } \\
60 \text { HIV negative pregnant } \\
\text { women] (Second half of } \\
\text { pregnancy) }\end{array}$ & SCID & EPDS & .817 & .690 & .780 \\
\hline Spies et al. (2009) [22] & South Africa & Prospective study & $\begin{array}{l}129 \text { pregnant women } \\
(<20 \text { weeks })\end{array}$ & SCID & K-10 & .660 & .730 & .540 \\
\hline \multirow[t]{2}{*}{ Stewart et al. (2013) [34] } & \multirow[t]{2}{*}{ Malawi } & \multirow[t]{2}{*}{ Validation study } & \multirow{2}{*}{$\begin{array}{l}224 \text { pregnant women } \\
\text { (28-34 weeks) }\end{array}$} & \multirow[t]{2}{*}{ SCID } & EPDS & .811 & .688 & .795 \\
\hline & & & & & SRQ & .833 & .763 & .813 \\
\hline
\end{tabular}

AUC area under curve, $B D I$ beck depression index, CES-D centre for epidemiologic studies depression scale, $C I$ confidence interval, $D S M-I V$ diagnostic and statistical manual of mental disorders version 4, EPDS Edinburgh postnatal depression scale, HAM-D Hamilton rating scale for depression, HSCL-25 Hopkins symptoms checklist 25, K-10 Kessler psychological distress scale 10, MINI mini-international neuropsychiatric interview, SCID structured clinical interviews for DSM IV axis 1 diagnoses, SRQ self-reporting questionnaire, Se sensitivity, Sp specificity, [ ] number in reference list, HIV human immunodeficiency virus

to .900. A forest plot showed that the included studies were heterogeneous because error bars for sensitivity and specificity plots did not include the summary valuessensitivity of .82 and specificity of .79 (Fig. 2). As such 5 distinct subgroups based on participants or type of instrument were formulated and graphical test using forest plots showed that one EPDS studies subgroup of all pregnant women was heterogeneous while other four were homogeneous (Figs. 3, 4 and 5). Schriger and colleagues recommended that a forest plot should consist of a minimum of two studies and discourages conducting heterogeneity tests when there are less than five studies [41].

\section{The EPDS}

The EPDS is a 10-item self-reported questionnaire about feelings of depression experienced in the postnatal period rated over the past 7 days with each item being rated on four exclusive scores that range from 0 to 3 [42]. The EPDS is shorter compared to other instruments (BDI, CES-D-20, HSCL-15 and SRQ) and takes about $5 \mathrm{~min}$ to complete.
The sensitivity and specificity of EPDS differed across studies which may be attributed to variations in study methodologies [43] and characteristics of populations under study [1]. The sensitivity of the EPDS across the 8 studies ranged from $\mathrm{Se}=.688$ to $\mathrm{Se}=1$, with a specificity from $\mathrm{Sp}=.733$ to $\mathrm{Sp}=.915$. EPDS had pooled sensitivity of. 80 and pooled specificity of .81 after excluding studies for pregnant women with Human Immunodeficiency Virus (HIV) [3] and those who were young [37, 39] (Fig. 3). Pooling was done in these two EPDS studies subgroups because they were considered to be sufficiently homogeneous in terms of participants, screening instrument and outcomes [44]. The EPDS had the highest level with an AUC ranging from .770 to .965 indicating a high level of accuracy in detecting depression in pregnant women in low resource settings.

\section{The BDI}

The BDI is a 21-item self-rating inventory which measures symptoms of depression on a scale from 0 to 3 [45]. Sensitivity of $\mathrm{BDI}$ in the two studies was $\mathrm{Se}=.867$ and $\mathrm{Se}=.82$ 


Study
BDI e Couto et al 2015
BDI Martins et al 2015
CES-D 20 Natamba et al 2014
EPDS Adewuya et al 2006
EPDS Alvarado-Esquivel et al 2014
EPDS Alvarado-Esquivel et al 2014 b
EPDS e Couto et al 2015
EPDS Fernandes et al 2011
EPDS Martins et al 2015
EPDS Rochat et al 2013
EPDS Stewart et al 2013
HAM-D e Couto et al 2015
HSCL-25 Kaaya et al 2002
K-10 Fernandes et al 2011
K-10 Spies et al 2009
SRQ Stewart et al 2013
Summary value

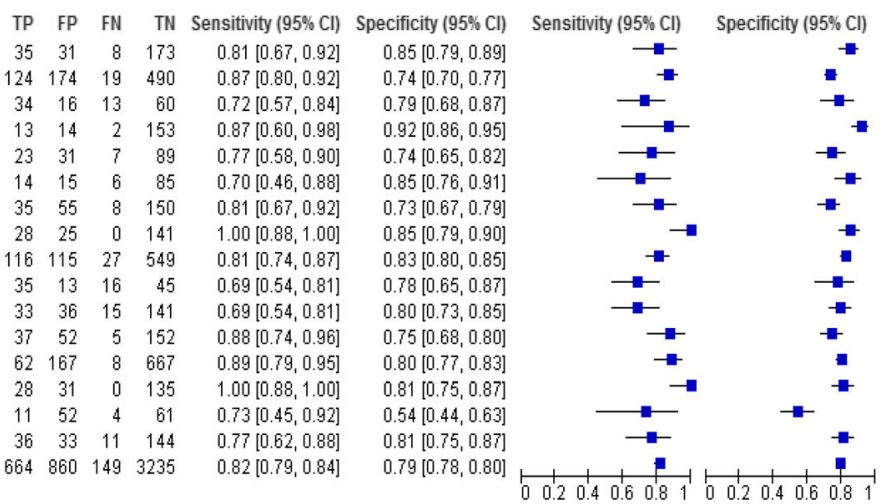

Fig. 2 Sensitivity and specificity of selected tools. BDI=Beck Depression Index, CES-D=Centre for Epidemiologic Studies Depression Scale, EPDS= Edinburgh Postnatal Depression Scale, HAM-D=Hamilton Rating Scale for Depression, HSCL 25=Hopkins Symptoms Checklist 25, K10=Kessler Psychological Distress Scale 10, SRQ 20=Self-Reporting Questionnaire 20, FN=False negative, FP=False positive, TN=True negative,

TP=True positive

with AUC of .87 and .90 respectively (Table 3) BDI had pooled $\mathrm{Se}=.85$ and pooled $\mathrm{Sp}=.76$ (Fig. 4).

\section{K-10}

The Kessler-10 (K-10) is a self-administered 10-item questionnaire which measures anxiety and depression rated over the past 4 weeks [46]. The data from the two K-10 studies were inconsistent with the second highest accuracy (AUC $=.95)$ in India and the lowest accuracy (AUC $=.66$ ) in South Africa and the highest sensitivity $(\mathrm{Se}=1.0)$ in India and lowest specificity $(\mathrm{Sp}=.54)$ in South Africa (pooled $\mathrm{Se}=.91$ and pooled $\mathrm{Sp}=.70)($ Fig. 5).

\section{Other instruments}

A number of other screening instruments were also reported as having been used in low resource settings. These were: CES-D, a 20 item self-rating scale which measures depressive symptomatology in the general population [47]; the HSCL-25, a self-report inventory for identifying common psychiatric symptoms [48] which include fifteen items for screening depression (HSCL15 ); the $S R Q$, a 20 item scale that is used to assess for psychiatric disturbance [49] and the HAM-D, a 21 items clinician administered scale that assesses severity of, and change in, depressive symptoms [50].

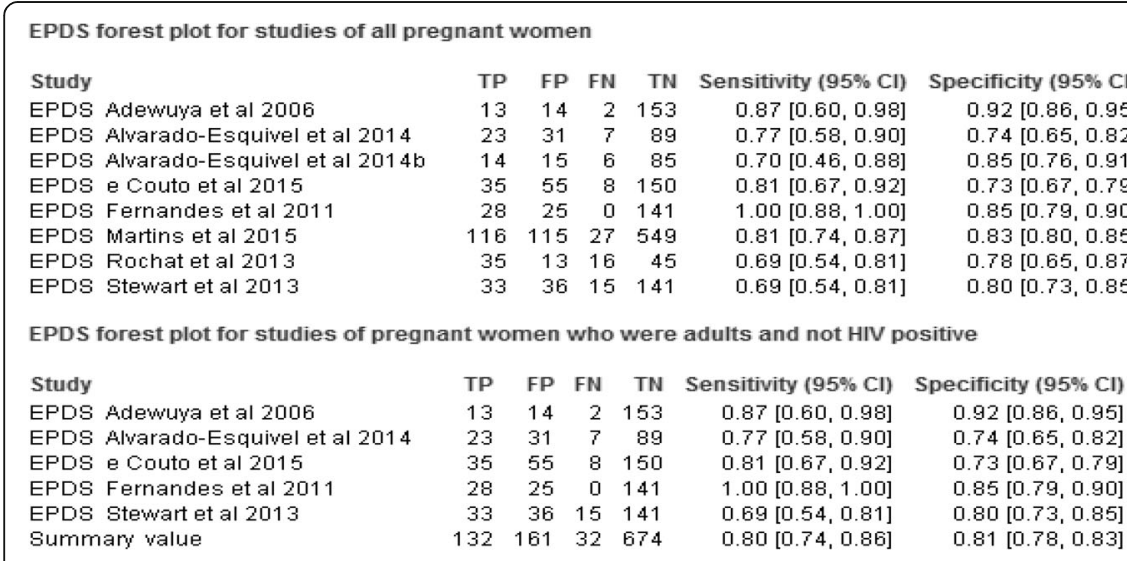

EPDS forest plot for studies of pregnant women who were HIV positive

$\begin{array}{lrrrrrr}\text { Study } & \text { TP } & \text { FP } & \text { FN } & \text { TN } & \text { Sensitivity }(95 \% \text { Cl) } & \text { Specificity }(95 \% \text { Cl) } \\ \text { EPDS Martins et al } 2015 & 116 & 115 & 27 & 549 & 0.81[0.74,0.87] & 0.83[0.80,0.85] \\ \text { EPDS Rochat et al 2013 } & 35 & 13 & 16 & 45 & 0.69[0.54,0.81] & 0.78[0.65,0.87] \\ \text { Summary value } & 151 & 128 & 43 & 594 & 0.78[0.71,0.83] & 0.82[0.79,0.85]\end{array}$

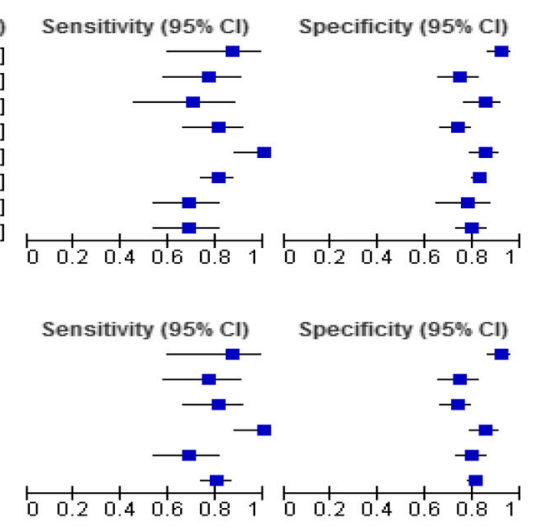

Fig. 3 Forest plot of EPDS studies. EPDS= Edinburgh Postnatal Depression Scale, FN=False negative, FP=False positive, TN=True negative, $\mathrm{TP}=$ True positive 


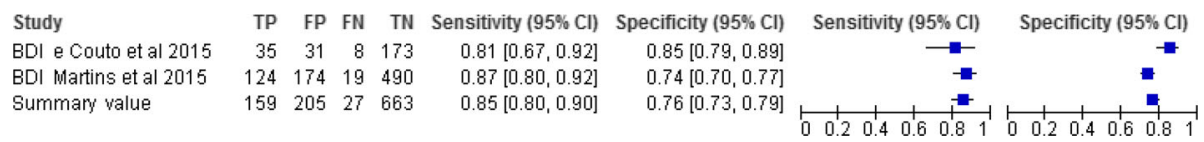

Fig. 4 Forest plot of $\mathrm{BDI}$ studies. $\mathrm{BDI}=\mathrm{Beck}$ Depression Index, $\mathrm{FN}=$ False negative, $\mathrm{FP}=$ False positive, $\mathrm{TN}=$ True negative, $T P=T$ rue positive

\section{Discussion}

An instrument being considered for selection for routine screening, should be inexpensive, be easy to administer, cause minimal discomfort and have high reliability and validity in distinguishing between cases and non-cases of a condition [51]. In this review, screening instruments with a pooled sensitivity/specificity balance $>85 \%$ were considered as ideal to distinguish between depressed and non-depressed women. The EPDS met criteria for both brevity and validity with this review, similar to two earlier systematic reviews [21, 24] which found high sensitivity, high specificity and the highest level of accuracy (AUC $=.965)$. Though the $\mathrm{K}-10$ had the best pooled sensitivity ( $\mathrm{Se}=.91)$, the EPDS had the best pooled specificity $(\mathrm{Sp}=.81)$. The BDI had a good sensitivity/ specificity balance $(\mathrm{Se}=.85$ and $\mathrm{Sp}=.76)$ respectively, but the EPDS sensitivity/specificity balance was more ideal with a higher specificity (important in screening out non-cases) and adequate sensitivity $(\mathrm{Se}=.80)$.

A second finding from this review is evidence that seven local language versions of depression screening instruments (BDI, CES-D-20, EPDS, HAM-D, HSCL25, K-10 and SRQ) had acceptable sensitivities or specificities and level of accuracy in antenatal clinics in low resource settings. However, none of these instruments were specifically designed to measure antenatal depression in low resource settings and their sensitivity and specificity varied with studies. The included studies had significant differences in methodology, population sampled, gestation period, type of instrument used and gold standards which indicated that there was clinical heterogeneity amongst included studies. Nevertheless, forest plots showed that distinct subgroups of studies which used similar participants and instruments were homogeneous. But one has to bear in mind that this method of identifying heterogeneity has limited power in detecting bias when studies are few [52].

It is documented that HIV prevalence in a population may influence the prevalence and severity of depression [3]. However, in this review, the instruments (EPDS and
K-10) which had highest sensitivity $(\mathrm{Se}=1.0)$ were validated in general population of pregnant women while lowest sensitivity ( $\mathrm{Se}=.69$ ) of EPDS was found in both general population of pregnant women, and in sample comprising of HIV positive and HIV negative pregnant women. In this review, it was clear that the pooled sensitivity of EPDS ( $\mathrm{Se}=.80$ ) for a subgroup of adult and non-HIV positive pregnant women was higher than that for HIV positive women $(\mathrm{Se}=.78)$. Nonetheless, one may not clearly ascertain from this review the extent to which HIV status of pregnant women influenced validity of screening instruments.

In this review, it was clear that in Mexico, sensitivity of EPDS among teenager pregnant women was 0.05 lower than its sensitivity among adult pregnant women $[36,37]$. This may suggest that the population sampled may influence validity of a screening instrument. Studies have found that instruments may have different levels of sensitivity and specificity when applied to women at different stages of pregnancy. In this review, the EPDS had both highest sensitivity $(\mathrm{Se}=1.0)[4]$ and lowest sensitivity $(\mathrm{Se}=.69)$ [34] among third trimester pregnant women and BDI had different sensitivity values among second trimester pregnant women in Brazil $[1,39]$. It was however not possible in this review it establish whether screening instruments may have different levels of sensitivity and specificity when applied to women at different stages of pregnancy due to inconsistencies in completeness of reporting in original studies.

Lastly, while systematic reviews are widely recognised as an efficient, reliable and comprehensive source of evidence for decision-making, few systematic reviews have considered effects on health equity [14]. In the light of this, the reviewers' recommendations were focused on the appropriate end-users (antenatal services in low resource settings) and we recognise that the findings are context-specific [14]. In this context, the EPDS emerged as the most suitable instrument for screening antenatal depression in low resource settings where time and other resources are limited. This performance of the

$$
\begin{array}{lrrrrrrrr}
\text { Study } & \text { TP } & \text { FP } & \text { FN } & \text { TN } & \text { Sensitivity }(95 \% \mathrm{Cl}) & \text { Specificity }(95 \% \mathrm{Cl}) & \text { Sensitivity }(95 \% \mathrm{Cl}) \\
\text { K-10 Fernandes et al } 2011 & 28 & 31 & 0 & 135 & 1.00[0.88,1.00] & 0.81[0.75,0.87] & & \text { Specificity (95\% Cl) } \\
\text { K-10 Spies et al 2009 } & 11 & 52 & 4 & 61 & 0.73[0.45,0.92] & 0.54[0.44,0.63] & &
\end{array}
$$

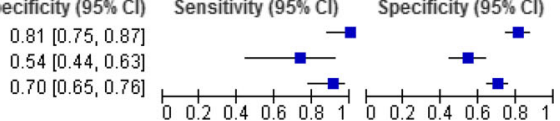

Fig. 5 Forest plot of K-10 studies. K10=Kessler Psychological Distress Scale 10, FN=False negative, FP=False positive, TN=True negative, TP=True positive 
EPDS in low resource settings is important as it supports the existing evidence from HICs which cannot always be applied effectively in low resource settings [53]. As such, this emic evidence will supplement the existing etic evidence to bring transformational health changes in antenatal care in low resource settings [13] which have heavy workloads, insufficient staff, poor funding and lack of medicines and supplies [11].

\section{Strengths and limitations}

One of the key strengths of the review is the specific evidence on screening tools used in antenatal services in low resource settings. It may serve as an efficient, reliable and comprehensive source of evidence for decisionmakers in low resource settings [14] since most evidence, generated from HICs, may not be applicable in low resource settings. A limitation of this review is that restrictions on language and date limits may have resulted in missing out some relevant articles.

\section{Conclusion}

This review suggests that the EPDS can be a suitable instrument of preference for screening antenatal depression in low resource settings because its level of accuracy ranged from moderate to high in various settings. The EPDS is an easy and cheap tool for clinicians to administer during antenatal attendances and can help in identifying pregnant women at risk of depression [39].

\section{Abbreviations}

AUC: Area under curve; BDI: Beck depression index; CES-D 20: Centre for epidemiologic studies depression scale 20; Cl: Confidence interval; CIDI: Composite international diagnostic interview; CINAHL: Cumulative index to nursing and allied health literature; DSM-IV: Diagnostic and statistical manual of mental disorders version 4; EPDS: Edinburgh postnatal depression scale; HAM-D: Hamilton rating scale for depression; HICs: High income countries; HIV: Human immunodeficiency virus; HSCL-15: Hopkins symptoms checklist 15; HSCL-25: Hopkins symptoms checklist 25; ICD-10: International classification of diseases version 10; K-10: Kessler psychological distress scale; LMICs: Low and middle income countries; MINI: Mini-international neuropsychiatric interview; PICOS: Participants index test comparator test outcome measures study setting; QUADAS: Quality assessment of diagnostic accuracy studies; SCID: Structured clinical interview for DSM-IV; SRQ: Self-reporting questionnaire; STARD: Standards for the reporting of diagnostic accuracy studies; UMICs: Upper middle income countries

\section{Acknowledgements}

We acknowledge all colleagues who offered guidance and technical support during development of the manuscript.

\section{Funding}

Funding for this review comes from a Doctor of Philosophy scholarship that was awarded to GC by University of Malawi through QZA-0484 NORHED 2013 grant. The funder did not play any part in the design of the study and collection, analysis, and interpretation of data and in writing the manuscript.

\section{Availability of data materials}

All data generated or analysed during this review are included in this manuscript and its supplementary information files.

\section{Authors' contributions}

GC drafted the manuscript under supervision of JC. GC designed protocol for the review with guidance from JC and both participated in each of its phases. GC conducted the search for articles. Both authors participated in the review and revision of the manuscript and have approved the final manuscript to be published.

\section{Competing interests}

The authors declare that they have no competing interests.

\section{Consent for publication}

Not applicable.

\section{Ethics approval and consent to participate}

This review is part of a doctoral project which was approved by the Senate Research Committee at the University of the Western Cape and College of Medicine Research and Ethics Committee at University of Malawi.

\section{Publisher's Note}

Springer Nature remains neutral with regard to jurisdictional claims in published maps and institutional affiliations.

\section{Author details}

${ }^{1}$ School of Nursing, University of the Western Cape, Private Bag X17, Bellville 7535, South Africa. ${ }^{2}$ Kamuzu College of Nursing, University of Malawi, Blantyre, Malawi. ${ }^{3}$ Honorary Affiliate, Sydney Nursing School, University of Sydney, Sydney, Australia.

Received: 24 September 2016 Accepted: 15 March 2017

Published online: 24 March 2017

\section{References}

1. e Couto TC, MMY B, Cardoso MN, Protzner AB, Garcia FD, Nicolato R, et al. What is the best tool for screening antenatal depression? J Affect Disord. 2015;178:12-7.

2. Stewart R, Umar E, Tomenson B, Creed F. A cross-sectional study of antenatal depression and associated factors in Malawi. Arch Womens Ment Health. 2014;17(2):145-54.

3. Rochat TJ, Tomlinson M, Newell M-L, Stein A. Detection of antenatal depression in rural HIV-affected populations with short and ultrashort versions of the Edinburgh Postnatal Depression Scale (EPDS). Arch Womens Ment Health. 2013;16(5):401-10.

4. Fernandes M, Srinivasan K, Stein A, Menezes G, Sumithra R, Ramchandani P. Assessing prenatal depression in the rural developing world: a comparison of two screening measures. Arch Womens Ment Health. 2011;14(3):209-16.

5. Faisal-Cury A, Menezes PR. Antenatal depression strongly predicts postnatal depression in primary health care. Rev Bras Psiquiatr. 2012;34(4):446-50.

6. Rahman A, Surkan PJ, Cayetano CE, Rwagatare P, Dickson KE. Grand Challenges: Integrating Maternal Mental Health into Maternal and Child Health Programmes. PLoS Med. 2013;10(5):1-7.

7. Lancaster CA, Gold KJ, Flynn HA, Yoo H, Marcus SM, Davis MM. Risk factors for depressive symptoms during pregnancy: a systematic review. Am J Obstet Gynecol. 2010;202(1):5-14.

8. Mathibe-Neke JM, Rothberg A, Langley G. The perception of midwives regarding psychosocial risk assessment during antenatal care. Health SA Gesondheid (Online). 2014;19(1):01-9.

9. Pilowsky DJ, Wu L-T. Screening instruments for substance use and brief interventions targeting adolescents in primary care: a literature review. Addict Behav. 2013:38(5):2146-53.

10. Bossuyt PM, Reitsma JB, Bruns DE, Gatsonis CA, Glasziou PP, Irwig L, et al. STARD 2015: an updated list of essential items for reporting diagnostic accuracy studies. Radiology. 2015;277(3):826-32.

11. Goldstuck ND. Healthcare in Low-resource Settings: the individual perspective. Healthcare in Low-resource Settings. 2014;2(2):4572.

12. World Bank World Bank list of economies. 2016. databank.worldbank.org/ data/download/site-content/CLASS.xls. Accessed 17 Mar 2017.

13. Lahariya C. Introducing Healthcare in Low-resource Settings. Healthcare Low-Resource Settings. 2013;1(1):1.

14. Welch VA, Petticrew M, O'Neill J, Waters E, Armstrong R, Bhutta ZA, et al. Health equity: evidence synthesis and knowledge translation methods. Syst Rev. 2013;2(1):1 
15. Trikalinos TA, Balion CM, Coleman Cl, Griffith L, Santaguida PL, Vandermeer B, et al. Meta-Analysis of Test Performance When There Is a "Gold Standard". J Gen Intern Med. 2012;27(1):56-66.

16. Pettersson A, Boström KB, Gustavsson P, Ekselius L. Which instruments to support diagnosis of depression have sufficient accuracy? A systematic review. Nord J Psychiatry. 2015;69(7):497-508.

17. Whiting P, Rutjes AW, Reitsma JB, Bossuyt PM, Kleijnen J. The development of QUADAS: a tool for the quality assessment of studies of diagnostic accuracy included in systematic reviews. BMC Med Res Methodol. 2003;3(1):25.

18. Radakovic R, Harley C, Abrahams S, Starr JM. A systematic review of the validity and reliability of apathy scales in neurodegenerative conditions. Int Psychogeriatr. 2015;27(06):903-23.

19. Onwuegbuzie AJ, Leech NL, Collins KM. Qualitative analysis techniques for the review of the literature. Qual Rep. 2012;17(28):1-28.

20. Austin MP, Lumley J. Antenatal screening for postnatal depression: a systematic review. Acta Psychiatr Scand. 2003;107(1):10-7.

21. Akena D, Joska J, Obuku EA, Amos T, Musisi S, Stein DJ. Comparing the accuracy of brief versus long depression screening instruments which have been validated in low and middle income countries: a systematic review. BMC Psychiatry. 2012;12(1):187.

22. Spies G, Stein D, Roos A, Faure S, Mostert J, Seedat S, et al. Validity of the Kessler 10 (K-10) in detecting DSM-IV defined mood and anxiety disorders among pregnant women. Arch Womens Ment Health. 2009;12(2):69-74.

23. Kaaya SF, Fawzi M, Mbwambo J, Lee B, Msamanga Gl, Fawzi W. Validity of the Hopkins Symptom Checklist-25 amongst HIV-positive pregnant women in Tanzania. Acta Psychiatr Scand. 2002;106(1):9-19.

24. Tsai AC, Scott JA, Hung KJ, Zhu JQ, Matthews LT, Psaros C, et al. Reliability and validity of instruments for assessing perinatal depression in African settings: systematic review and meta-analysis. PLoS One. 2013;8(12):e82521.

25. Adewuya AO, Ola BA, Dada AO, Fasoto OO. Validation of the Edinburgh Postnatal Depression Scale as a screening tool for depression in late pregnancy among Nigerian women. J Psychosom Obstet Gynaecol. 2006;27(4):267-72.

26 Rochat TJ. Depression among pregnant women testing for HIV in rura South Africa. Doctoral Thesis. Stellenbosch: University of Stellenbosch; 2011.

27 Abiodun O. A validity study of the Hospital Anxiety and Depression Scale in general hospital units and a community sample in Nigeria. Br J Psychiatry. 1994;165(5):669-72.

28 Abiodun O, Adetoro O, Ogunbode O. Psychiatric morbidity in a pregnant population in Nigeria. Gen Hosp Psychiatry. 1993;15(2):125-8.

29 Aderibigbe Y, Gureje O. The validity of the 28-item General Health Questionnaire in a Nigerian antenatal clinic. Soc Psychiatry Psychiatr Epidemiol. 1992;27(6):280-3.

30 Nhiwatiwa S, Patel V, Acuda W. Predicting postnatal mental disorder with a screening questionnaire: a prospective cohort study from Zimbabwe. J Epidemiol Community Health. 1998;52(4):262-6.

31 Adewuya AO, Ola BA, Aloba OO, Dada AO, Fasoto OO. Prevalence and correlates of depression in late pregnancy among Nigerian women. Depress Anxiety. 2007:24(1):15-21.

32 Tsai A, Tomlinson M, Dewing S, Roux I, Harwood J, Chopra M, et al. Antenatal depression case finding by community health workers in South Africa: feasibility of a mobile phone application. Arch Womens Ment Health. 2014;17(5):423-31.

33 Vythilingum B, Field S, Kafaar Z, Baron E, Stein D, Sanders L, et al. Screening and pathways to maternal mental health care in a South African antenatal setting. Arch Womens Ment Health. 2013;16(5):371-9.

34 Stewart R, Umar E, Tomenson B, Creed F. Validation of screening tools for antenatal depression in Malawi-A comparison of the Edinburgh Postnatal Depression Scale and Self Reporting Questionnaire. J Affect Disord. 2013; 150(3):1041-7.

35 Natamba BK, Achan J, Arbach A, Oyok TO, Ghosh S, Mehta S, et al. Reliability and validity of the center for epidemiologic studies-depression scale in screening for depression among HIV-infected and-uninfected pregnant women attending antenatal services in northern Uganda: a cross-sectional study. BMC Psychiatry. 2014;14(1):1.

36 Alvarado-Esquivel C, Sifuentes-Alvarez A, Salas-Martinez C. Validation of the Edinburgh postpartum depression scale in a population of adult pregnant women in Mexico. J Clin. Med. Res. 2014;6(5):374.

37 Alvarado-Esquivel C, Sifuentes-Alvarez A, Salas-Martinez C. The use of the edinburgh postpartum depression scale in a population of teenager pregnant women in Mexico: a validation study. Clin Pract Epidemiol Ment Health. 2014;10:129-32.

38 Spies G, Stein D, Roos A, Faure S, Mostert J, Seedat S, et al. Validity of the Kessler 10 (K-10) in detecting DSM-IV defined mood and anxiety disorders among pregnant women. Archives of women's mental health. 2009;12(2):69-74

39 Martins Cde S, Motta JV, Quevedo LA, Matos MB, Pinheiro KA, Souza LD, et al. Comparison of two instruments to track depression symptoms during pregnancy in a sample of pregnant teenagers in Southern Brazil. J Affect Disord. 2015;177:95-100

40 Fischer JE, Bachmann LM, Jaeschke $\mathrm{R}$. A readers' guide to the interpretation of diagnostic test properties: clinical example of sepsis. Intensive Care Med. 2003:29(7):1043-51.

41 Schriger DL, Altman DG, Vetter JA, Heafner T, Moher D. Forest plots in reports of systematic reviews: a cross-sectional study reviewing current practice. Int J Epidemiol. 2010;39(2):421-9.

42 Tran TD, Tran T, La B, Lee D, Rosenthal D, Fisher J. Screening for perinatal common mental disorders in women in the north of Vietnam: a comparison of three psychometric instruments. J Affect Disord. 2011;133(1):281-93.

43 Gibson J, McKenzie-McHarg K, Shakespeare J, Price J, Gray R. A systematic review of studies validating the Edinburgh Postnatal Depression Scale in antepartum and postpartum women. Acta Psychiatr Scand. 2009:119(5):350-64.

44 Higgins JP, Green S. Cochrane handbook for systematic reviews of interventions. Version 5.1.0 [updated March 2011]. The Cochrane Collaboration, 2011, vol. 4. Chichester: Wiley; 2011.

45 Beck AT, Ward C, Mendelson M. Beck depression inventory (BDI). Arch Gen Psychiatry. 1961;4(6):561-71.

46 Kessler R, Mroczek D. Kessler psychological distress scale (K10). Boston: Harvard Medical School; 1996.

47 Radloff LS. The CES-D scale a self-report depression scale for research in the general population. Appl Psychol Meas. 1977;1(3):385-401.

48 Derogatis LR, Lipman RS, Rickels K, Uhlenhuth EH, Covi L. The Hopkins Symptom Checklist (HSCL): A self-report symptom inventory. Behav Sci. 1974;19(1):1-15

49 WHO. A user's guide to the Self Reporting Questionnaire (SRQ). Geneva: World Health Organization; 1994.

50 Hamilton M. A rating scale for depression. J Neurol Neurosurg Psychiatry. 1960;23(1):56

51 Zhu W, Zeng N, Wang N. Sensitivity, specificity, accuracy, associated confidence interval and ROC analysis with practical SAS ${ }^{\circledR}$ implementations. 2010:1-9.

52 Dinnes J, Deeks J, Kirby J, Roderick P. A methodological review of how heterogeneity has been examined in systematic reviews of diagnostic test accuracy. Health Technol Assess. 2005;9(12):1-128.

53 BOLDER Research Group. Better Outcomes through Learning, Data, Engagement, and Research (BOLDER)-a system for improving evidence and clinical practice in low and middle income countries. F1000Research. 2016;5:693.

\section{Submit your next manuscript to BioMed Central and we will help you at every step:}

- We accept pre-submission inquiries

- Our selector tool helps you to find the most relevant journal

- We provide round the clock customer support

- Convenient online submission

- Thorough peer review

- Inclusion in PubMed and all major indexing services

- Maximum visibility for your research

Submit your manuscript at www.biomedcentral.com/submit 